\title{
ON THE FINITENESS OF THE NUMBER OF EIGENVALUES OF JACOBI OPERATORS BELOW THE ESSENTIAL SPECTRUM
}

\author{
FRANZ LUEF AND GERALD TESCHL
}

\begin{abstract}
We present a new oscillation criterion to determine whether the number of eigenvalues below the essential spectrum of a given Jacobi operator is finite or not. As an application we show that Kenser's criterion for Jacobi operators follows as a special case.
\end{abstract}

\section{INTRODUCTION}

The goal of the present paper is to determine whether the number of eigenvalues below the essential spectrum of the Jacobi operator on $\ell^{2}(\mathbb{N})$ associated with

$$
(\tau f)(n)=a(n) f(n+1)+a(n-1) f(n-1)-b(n) f(n),
$$

where

$$
a(n) \in \mathbb{R} \backslash\{0\}, \quad b(n) \in \mathbb{R}, \quad n \in \mathbb{N},
$$

is finite or not.

We will assume $a(n)<0$ (which is no restriction by [15, Lemma 1.6). One of the main cases of interest is $a(n)=-1$ and one usually starts with the operator $H_{0}$ associated with $b_{0}(n)=2$. The spectrum is given by $\sigma\left(H_{0}\right)=[0,4]$. In particular, there are no eigenvalues below the essential spectrum. Perturbing $b_{0}$ we can add any finite number of eigenvalues even if our perturbation is of compact support. However, the question is, can we at least determine whether the number of eigenvalues is finite or not, by looking at the asymptotics of the perturbation? Moreover, what is the precise asymptotics separating the two cases?

The natural tool for investigating such questions is oscillation theory since finiteness of the number of eigenvalues is equivalent to the operator being nonoscillatory. This fact first appeared in [5]. The precise relation between the number of eigenvalues and the number of nodes was established only recently by one of us in 14 .

In the case of Sturm-Liouville operators there is a famous theorem by Kneser [10] which gives a simple and beautiful answer to this question, with many subsequent extensions by others. The most recent one being by [4, who give a unified result containing all previously known ones as special cases.

1991 Mathematics Subject Classification. Primary 36A10, 39A70; Secondary 34B24, 34L05.

Key words and phrases. Discrete oscillation theory, Jacobi operators, spectral theory, Kneser's theorem.

J. Difference Equ. Appl. 10, no. 3, 299-307 (2004). 
Unfortunately, it is not possible to use the same proof in the discrete case. To understand why, let us first review the proof of Kneser's theorem in the SturmLiouville case. The key idea is that the equation

$$
\tau_{0}=-\frac{d^{2}}{d x^{2}}+\frac{\mu}{x^{2}}
$$

is of Euler type. Hence it is explicitly solvable with a fundamental system given by

$$
x^{\frac{1}{2} \pm \sqrt{\mu+\frac{1}{4}}} \text {. }
$$

There are two cases to distinguish. If $\mu \geq-1 / 4$ all solutions are nonoscillatory. If $\mu<-1 / 4$ one has to take real/imaginary parts and all solutions are oscillatory. Hence a straightforward application of Sturm's comparison theorem between $\tau_{0}$ and

$$
\tau=-\frac{d^{2}}{d x^{2}}+q(x)
$$

yields

$$
\lim _{x \rightarrow \infty} \inf _{\sup }\left(x^{2} q(x)\right) \underset{<}{>}-\frac{1}{4} \text { implies } \begin{gathered}
\text { nonoscillation } \\
\text { oscillation }
\end{gathered} \text { of } \tau \text { near } \infty .
$$

Since Sturm's comparison theorem is also available for Jacobi operators (see, e.g, [15], Lemma 4.4) it seems easy to generalize this result by considering the discrete Euler equation

$$
u(n+1)-2 u(n)+u(n-1)-\frac{\mu}{n(n-1)} u(n-1)=0 .
$$

However, unfortunately, this equation is not symmetric! The corresponding results for this equation can be found as special cases in [1], Section 6.11. Thus a straightforward generalization is not possible.

One approach is to consider generalized ordinary differential expressions which contain difference equations as a special case. This can be found in [5] and 11] (see Section 2.2). As an alternative, we will prove a new oscillation criterion and show that Kneser's criterion follows as a special case.

For further (non-)oscillation criteria we refer to Hinton and Lewis [7] and Hooker and Patula [8] (see also [6], 9], [12], and [13]). For a different approach using Birman-Schwinger type arguments see [2, 3].

Our present paper was motivated by the work of Gesztesy and Ünal 4 mentioned earlier. In fact, it can be viewed as a discrete generalization of their results. However, again a straightforward generalization is not possible since their proofs also rely on explicit solubility of the involved equations.

\section{MAin Results AND APPLICATIONS}

Before we can write down our main result, we need to fix some notation. Recall that $\tau$ is called oscillatory if one (and hence any) real-valued solution of $\tau u=0$ has an infinite number of nodes, that is, points $n \in \mathbb{N}$, such that either

$$
u(n)=0 \quad \text { or } \quad a(n) u(n) u(n+1)>0 .
$$

In the special case $a(n)<0, n \in \mathbb{N}$, a node of $u$ is precisely a sign flip of $u$ as one would expect. In the general case, however, one has to take the sign of $a(n)$ into account. 
Recall that if $u_{0}(n)>0$ solves

$$
\left(\tau_{0} u\right)(n)=a(n) u_{0}(n+1)+a(n-1) u_{0}(n-1)-b_{0}(n) u_{0}(n)=0,
$$

then

$$
\hat{u}_{0}(n)=u_{0}(n) Q_{0}(n), \quad Q_{0}(n)=\sum_{j=0}^{n-1} \frac{-1}{a(j) u_{0}(j) u_{0}(j+1)},
$$

is a second, linearly independent positive solution. A positive solution is called minimal if

$$
\lim _{n \rightarrow \infty} Q_{0}(n)=\infty
$$

Minimal solutions are unique up to a multiple. See 15, Section 2.3 for more information. With this notation our main result reads as follows:

Theorem 2.1. Suppose $a(n) \in \mathbb{R} \backslash\{0\}, b(n), b_{0}(n) \in \mathbb{R}$ such that $a_{0}<|a(n)|<A_{0}$ for some real constants $0<a_{0}<A_{0}$ and let $u_{0}$ be a non-decreasing minimal positive solution of $\tau_{0} u_{0}=0$ (as defined in (2.2)). Introduce

$$
A(n)=\frac{2 a(n-1) a(n+1)}{a(n-1)+a(n+1)}, \quad n \in \mathbb{N},
$$

and

$$
Q_{0}(n)=\sum_{j=0}^{n-1} \frac{-1}{a(j) u_{0}(j) u_{0}(j+1)}, \quad n \in \mathbb{N} .
$$

Then $\tau$ is nonoscillatory if

$$
\liminf _{n \rightarrow \infty}-A(n) u_{0}^{4}(n) Q_{0}^{2}(n)\left(b(n)-b_{0}(n)\right)>-\frac{1}{4}
$$

and oscillatory if

$$
\limsup _{n \rightarrow \infty}-A(n) u_{0}^{4}(n) Q_{0}^{2}(n)\left(b(n)-b_{0}(n)\right)<-\frac{1}{4} .
$$

The proof will be given in Section 3 below.

As a first application, let us show how this result can be used to answer our question posed in the introduction. We choose

$$
a(n)=-1, \quad b_{0}(n)=2 .
$$

Then we have

$$
u_{0}(n)=1 \quad \text { and } \quad \hat{u}_{0}(n)=n
$$

and thus

(2.11) $\lim _{n \rightarrow \infty} \inf _{\sup }\left(n^{2}(b(n)-2)\right) \underset{<}{>}-\frac{1}{4}$ implies $\begin{gathered}\text { nonoscillation } \\ \text { oscillation }\end{gathered}$ of $\tau$ near $\infty$,

which is the claimed generalization of Kneser's result. Clearly, the next question is what happens in the limiting case, where $\lim _{n \rightarrow \infty} n^{2}(b(n)-2)=-4^{-1}$ ? This can be answered by our result as well:

Recall the iterated logarithm

$$
\ln _{0}(x)=x, \quad \ln _{k}(x)=\ln _{k-1}(\ln (x)),
$$

where $\ln _{k}(x)$ is defined for $x>\mathrm{e}_{k}$, with $\mathrm{e}_{1}=0, \mathrm{e}_{k}=\mathrm{e}^{\mathrm{e}_{k-1}}$. 
Corollary 2.2. Let

$$
a(n)=-1, \quad b_{k}(n)=2-\frac{1}{4} \sum_{j=0}^{k-1} \frac{1}{\prod_{\ell=0}^{j} \ln _{\ell}(n)^{2}} .
$$

Then $\tau$ is nonoscillatory if

$$
\liminf _{n \rightarrow \infty}\left(\prod_{j=0}^{k} \ln _{j}(n)\right)^{2}\left(b(n)-b_{k}(n)\right)>-\frac{1}{4}
$$

and oscillatory if

$$
\limsup _{n \rightarrow \infty}\left(\prod_{j=0}^{k} \ln _{j}(n)\right)^{2}\left(b(n)-b_{k}(n)\right)<-\frac{1}{4} .
$$

Proof. To show how this follows from our result we consider

$$
u_{k}(n)=\sqrt{\prod_{j=0}^{k-1} \ln _{j}(n)}
$$

which is a solution of $\tilde{\tau}_{k}$ associated with

$$
a(n)=-1, \quad \tilde{b}_{k}(n)=\frac{u_{k}(n+1)+u_{k}(n-1)}{u_{k}(n)} .
$$

To prove the claim it suffices to show

$$
\begin{aligned}
\tilde{b}_{k}(n) & =b_{k}(n)+O\left(n^{-3}\right), \\
Q_{k}(n) & =\ln _{k}(n)+O(1)
\end{aligned}
$$

since the differences will not contribute to the limits from above.

To establish (2.18) we first recall the following formulas for the first and second derivative of $\ln _{k}(x)$ :

$$
\begin{aligned}
& \ln _{k}^{\prime}(x)=\prod_{j=0}^{k-1} \frac{1}{\ln _{j}(x)} \\
& \ln _{k}^{\prime \prime}(x)=-\ln _{k}^{\prime}(x) \sum_{j=1}^{k} \ln _{j}^{\prime}(x), \quad x>\mathrm{e}_{k} .
\end{aligned}
$$

Now we can show (2.18). First of all we have

$$
Q_{k}(n)=\int^{n} \frac{d x}{u_{k}(x)^{2}}+O(1)=\ln _{k}(n)+O(1) .
$$


The second claim is a bit harder. We begin with

$$
\begin{aligned}
\frac{u_{k}(n \pm 1)}{u_{k}(n)}= & \left(\prod_{j=0}^{k-1} \frac{\ln _{j}(n \pm 1)}{\ln _{j}(n)}\right)^{1 / 2}=\prod_{j=0}^{k-1}\left(\sum_{\ell=0}^{\infty} \frac{(-1)^{\ell}}{\ell !} \frac{\ln _{j}^{(\ell)}(n)}{\ln _{j}(n)}\right)^{1 / 2} \\
= & \prod_{j=0}^{k-1}\left(1 \pm \frac{\ln _{j}^{\prime}(n)}{\ln _{j}(n)}+\frac{1}{2} \frac{\ln _{j}^{\prime \prime}(n)}{\ln _{j}(n)}+O\left(n^{-3}\right)\right)^{1 / 2} \\
= & \prod_{j=0}^{k-1}\left(1 \pm \frac{1}{2} \ln _{j+1}^{\prime}(n)+\frac{1}{4}\left(\frac{\ln _{j}^{\prime \prime}(n)}{\ln _{j}(n)}-\frac{\ln _{j+1}^{\prime}(n)^{2}}{2}\right)+O\left(n^{-3}\right)\right) \\
= & 1 \pm \frac{1}{2} \sum_{j=0}^{k-1} \ln _{j+1}^{\prime}(n)+\frac{1}{4} \sum_{j=0}^{k-1}\left(\frac{\ln _{j}^{\prime \prime}(n)}{\ln _{j}(n)}-\frac{\ln _{j+1}^{\prime}(n)^{2}}{2}\right)+ \\
& +\frac{1}{4} \sum_{j=0}^{k-1} \ln _{j+1}^{\prime}(n) \sum_{\ell=0}^{j-1} \ln _{\ell+1}^{\prime}(n)+O\left(n^{-3}\right) . \\
= & 1 \pm \frac{1}{2} \sum_{j=0}^{k-1} \ln _{j+1}^{\prime}(n)-\frac{1}{8} \sum_{j=0}^{k-1} \ln _{j+1}^{\prime}(n)^{2}+O\left(n^{-3}\right) .
\end{aligned}
$$

Now combining both formulas we obtain the desired result

$$
\tilde{b}_{k}(n)=2-\frac{1}{4} \sum_{j=0}^{k-1} \ln _{j+1}^{\prime}(n)^{2}+O\left(n^{-3}\right)=b_{k}(n)+O\left(n^{-3}\right) .
$$

Another interesting example is the case

$$
b_{0}(n)=-a(n)-a(n-1) .
$$

Again we can take $u_{0}(n)=1$ to obtain

Corollary 2.3. Let $a_{0} \leq|a(n)| \leq A_{0}$ and abbreviate

$$
A(n)=\frac{2 a(n-1) a(n+1)}{a(n-1)+a(n+1)}, \quad Q_{0}(n)=\sum_{j=0}^{n-1} \frac{-1}{a(j)} .
$$

Then $\tau$ is nonoscillatory if

$$
\liminf _{n \rightarrow \infty}-A(n) Q_{0}(n)^{2}(b(n)+a(n-1)+a(n))>-\frac{1}{4}
$$

and oscillatory if

$$
\limsup _{n \rightarrow \infty}-A(n) Q_{0}(n)^{2}(b(n)+a(n-1)+a(n))<-\frac{1}{4} .
$$

Of course one could take two arbitrary sequence $a(n)<0$ and $u_{0}(n)>0$ such that $u_{0}$ is non-decreasing and (2.4) is satisfied, compute $b_{0}(n)=-\left(a(n) u_{0}(n+1)+\right.$ $\left.a(n-1) u_{0}(n-1)\right) / u_{0}(n)$, and apply Theorem 2.1 to get a new (non)oscillation criterion. 


\section{Proof of Theorem 2.1}

Suppose $a(n) \in \mathbb{R} \backslash\{0\}, b(n), b_{0}(n) \in \mathbb{R}$ such that

$$
a_{0} \leq|a(n)| \leq A_{0}
$$

for some real constants $0<a_{0}<A_{0}$ and let $u_{0}$ be a non-decreasing minimal positive solution of $\tau_{0} u_{0}=0$ as in the previous section. Note that the corresponding second positive solution $\hat{u}_{0}(n)$ is increasing.

First we collect some basic facts which will be needed later on.

Lemma 3.1. Let $u_{0}$ be a minimal positive non-decreasing solution, then we have

$$
\lim _{n \rightarrow \infty} \frac{u_{0}(n+1)}{u_{0}(n)}=\lim _{n \rightarrow \infty} \frac{u_{0}(n)}{u_{0}(n-1)}=1
$$

and

$$
u_{0}(n) \hat{u}_{0}(n)=u_{0}^{2}(n) Q_{0}(n) \geq \frac{n}{A_{0}} .
$$

Proof. Monotonicity of $u_{0}$ implies

$$
\frac{1}{u_{0}(j+1)^{2}} \leq \frac{1}{u_{0}(j) u_{0}(j+1)} \leq \frac{1}{u_{0}(j)^{2}}
$$

Summing the last expression from 0 to $n-1$ and subtracting the right side yields

$$
0 \leq \sum_{j=0}^{n-1} \frac{1}{u_{0}(j) u_{0}(j+1)}\left(\frac{u_{0}(j+1)}{u_{0}(j)}-1\right) \leq \frac{1}{u_{0}(0)^{2}}-\frac{1}{u_{0}(n)^{2}} \leq \frac{1}{u_{0}(0)^{2}} .
$$

Since $u(n)$ is minimal,

$$
\sum_{j=0}^{n-1} \frac{1}{u_{0}(j) u_{0}(j+1)} \geq a_{0} Q_{0}(n) \rightarrow \infty
$$

implies the first result.

For the second claim we use

$$
Q_{0}(n)=\sum_{j=0}^{n-1} \frac{-1}{a(j) u_{0}(j) u_{0}(j+1)} \geq \frac{1}{A_{0}} \sum_{j=0}^{n-1} \frac{1}{u_{0}(j+1)^{2}} \geq \frac{n}{A_{0} u_{0}(n)^{2}}
$$

finishing the proof.

Our next goal is to find a suitable comparison equation. We do this by trying the ansatz

$$
u_{1}(n)=u_{0}(n) Q_{0}(n)^{\alpha} .
$$

Then $u_{1}(n)$ satisfies

$$
\tau_{1} u(n)=a(n) u_{1}(n+1)+a(n-1) u_{1}(n-1)+b_{1}(n) u_{1}(n)=0
$$

with $b_{1}(n)$ given by

$$
\begin{aligned}
b_{1}(n)= & -\frac{a(n) u_{0}(n+1)}{u_{0}(n)}\left(1-\frac{1}{a(n) u_{0}(n+1)^{2} Q_{0}(n)}\right)^{\alpha} \\
& -\frac{a(n-1) u_{0}(n-1)}{u_{0}(n)}\left(1+\frac{1}{a(n-1) u_{0}(n-1)^{2} Q_{0}(n)}\right)^{\alpha} .
\end{aligned}
$$

In order to get an oscillating comparison equation we need to admit $\alpha \in \mathbb{C}$. However, this will also render $b_{1}(n)$ complex and hence it will be of no use for us. 
To overcome this problem we look at the asymptotic behavior of $b_{1}(n)$ for $n \rightarrow \infty$, which is given by

$$
b_{1}(n)=b_{0}(n)+\mu U(n)+O\left(\frac{1}{u_{0}^{6}(n) Q_{0}^{3}(n)}\right), \quad \mu=\alpha(\alpha-1),
$$

where

$$
U(n)=\frac{1}{2 u_{0}^{4}(n) Q_{0}^{2}(n)}\left(\frac{-u_{0}(n)}{a(n+1) u_{0}(n+1)}+\frac{-u_{0}(n)}{a(n-1) u_{0}(n-1)}\right) .
$$

If $\alpha \in \mathbb{R}$ we can choose $b_{1}(n)$ directly as comparison potential to obtain that $\tau$ is nonoscillatory if

$$
\liminf _{n \rightarrow \infty} \frac{b(n)-b_{0}(n)}{b_{1}(n)-b_{0}(n)}>\mu \text {. }
$$

Using the optimal value $\alpha=\frac{1}{2}$ plus the expansion from above we end up with

$$
\liminf _{n \rightarrow \infty} \frac{b(n)-b_{0}(n)}{U(n)}>-\frac{1}{4}
$$

This settles the first part of our theorem. Now we come to the harder one. As already noticed, in order to get an oscillating comparison equation we need to choose complex values for $\alpha$. Our strategy is to choose $\alpha=\frac{1}{2}+\mathrm{i} \varepsilon$ such that at least $\mu=-\frac{1}{4}-\varepsilon^{2}$ remains real and take $\tilde{b}_{1}(n)=b_{0}(n)+\mu U(n)$ as comparison equation. Of course we do not know the solutions of this equation, but our hope is that they are asymptotically given by the real/imaginary parts of

$$
u_{1}(n)=u_{0}(n) \sqrt{Q_{0}(n)}\left(\cos \left(\varepsilon \ln Q_{0}(n)\right)+\mathrm{i} \sin \left(\varepsilon \ln Q_{0}(n)\right)\right) .
$$

Hence if we can show that there are solutions $\tilde{u}_{1}$ of $\tilde{\tau}_{1} \tilde{u}_{1}=0$ satisfying

$$
\tilde{u}_{1}(n)=u_{1}(n)(1+o(1))
$$

we are done.

To show this, we begin with

$$
\tau_{1} \tilde{u}_{1}(n)=\Delta(n) \tilde{u}_{1}(n), \quad \Delta(n)=b_{1}(n)-\tilde{b}_{1}(n),
$$

and use the solution formula for the inhomogeneous equation ([15, eqn. (1.48)) to obtain the following equation

$$
\tilde{u}_{1}(n)=u_{1}(n)-\sum_{j=n+1}^{n_{0}} u_{1}(n) u_{1}(j)\left(Q_{1}(n)-Q_{1}(j)\right) \Delta(j) \tilde{u}_{1}(j),
$$

where $Q_{1}$ is defined as $Q_{0}$ but with $u_{1}$ in place of $u_{0}$. Formally, letting $n_{0} \rightarrow \infty$, and setting

$$
\tilde{u}_{1}(n)=u_{1}(n) v(n)
$$

we obtain

$$
v(n)=1-\sum_{j=n+1}^{\infty} u_{1}(j)^{2}\left(Q_{1}(n)-Q_{1}(j)\right) \Delta(j) v(j) .
$$


If we can show existence of a solution $v(n)=1+o(1)$ of this last equation, we are done. For this it suffices to verify the assumptions of [15], Lemma 7.8. Hence we need to estimate the kernel of the above sum equation. Using

$$
\begin{aligned}
\left|Q_{1}(n)-Q_{1}(j)\right| & \leq \sum_{k=n}^{j} \frac{1}{a(k) u_{0}(k) u_{0}(k+1) \sqrt{Q_{0}(k) Q_{0}(k+1)}} \\
& \leq \frac{1}{a_{0}} \sum_{k=n}^{j} \frac{1}{u_{0}(k)^{2} Q_{0}(k)}
\end{aligned}
$$

and

$$
|\Delta(j)| \leq \frac{\text { const }}{u_{0}(j)^{6} Q_{0}(j)^{3}}
$$

we obtain by Lemma 3.1

$$
\begin{aligned}
\left|u_{1}(j)^{2}\left(Q_{1}(n)-Q_{1}(j)\right) \Delta(j)\right| & \leq \frac{\text { const }}{u_{0}(j)^{4} Q_{0}(j)^{2}} \sum_{k=n}^{j} \frac{1}{u_{0}(k)^{2} Q_{0}(k)} \\
& \leq \text { const } \frac{\ln (j)}{j^{2}} .
\end{aligned}
$$

Thus we can apply [15], Lemma 7.8 to conclude existence of a solution of type (3.16) which finishes the proof of Theorem 2.1.

\section{REFERENCES}

[1] R. P. Agarwal, Difference Equations and Inequalities. Theory, Methods, and Applications, 2nd ed., Marcel Dekker, New York, 2000.

[2] J. S. Geronimo, On the spectra of infinite-dimensional Jacobi matrices, J. App. Th., 53, 251-265 (1988).

[3] J. S. Geronimo, An upper bound on the number of eigenvalues of an infinite-dimensional Jacobi matrix, J. Math. Phys., 23, 9171982.

[4] F. Gesztesy and M. Ünal, Perturbative oscillation criteria and Hardy-type inequalities, Math. Nachr. 189, 121-144 (1998).

[5] I. M. Glazman, Direct Methods of Qualitative Spectral Analysis of Singular Differential Operators, I.P.S.T., Jerusalem, 1965.

[6] P. Hartman, Difference equations: Disconjugacy, principal solutions, Green's functions, complete monotonicity, Trans. Amer. Math. Soc., 246, 1-30 (1978).

[7] D. B. Hinton and R. T. Lewis, Spectral analysis of second order difference equations, J. Math. Anal. Appl. 63, 421-438 (1978).

[8] J. W. Hooker and W. T. Patula, Riccati type transformations for second-order linear difference equations, J. Math. Anal. Appl. 82, 451-462 (1981).

[9] J. W. Hooker, M. K. Kwong and W. T. Patula, Oscillatory second-order linear difference equations and Riccati equations, SIAM J. Math. Anal., 18, 54-63 (1987).

[10] A. Kneser, Untersuchungen über die reellen Nullstellen der Integrale linearer Differentialgleichungen, Math. Ann. 42, 409-435 (1893).

[11] A.B. Mingarelli, Volterra-Stieltjes Integral Equations and Generalized Ordinary Differential Expressions, Lecture Notes in Mathematics 989, Springer, Berlin, 1983.

[12] W. T. Patula, Growth and oscillation properties of second order linear difference equations, SIAM J. Math. Anal. 6, 55-61 (1979).

[13] W. T. Patula, Growth, oscillation and comparison theorems for second order linear difference equations, SIAM J. Math. Anal. 6, 1272-1279 (1979).

[14] G. Teschl, Oscillation theory and renormalized oscillation theory for Jacobi operators, J. Diff. Eqs. 129, 532-558 (1996).

[15] G. Teschl, Jacobi Operators and Completely Integrable Nonlinear Lattices, Math. Surv. and Mon. 72, Amer. Math. Soc., Rhode Island, 2000. 
Institut für Mathematik, Strudlhofgasse 4, 1090 Wien, Austria, and International Erwin Schrödinger Institute for Mathematical Physics, Boltzmanngasse 9, 1090 Wien, Austria

Institut für Mathematik, Strudlhofgasse 4, 1090 Wien, Austria, and International Erwin Schrödinger Institute for Mathematical Physics, Boltzmanngasse 9, 1090 Wien, Austria

E-mail address: Gerald.Teschl@univie.ac.at

URL: http://www.mat.univie.ac.at/〜 gerald/ 